\section{Orphan products: an emerging trend in drug approvals}

\author{
Timothy Coté, Aditya Kelkar, Kui Xu, M. Miles Braun and M. Ian Phillips
}

As highlighted in a recent News Feature, Rare incentives (Nature Rev. Drug Discov. $7,190-191 ; 2008)^{1}, 2008$ saw the $25^{\text {th }}$ anniversary of the US Orphan Drug Act (ODA), which was promulgated in recognition of the economic barriers to developing drugs for rare diseases (defined as those that affect fewer than 200,000 persons in the United States) ${ }^{2}$. The ODA encourages the development of promising new compounds by providing significant fiscal benefits: FDA-enforced marketing exclusivity, tax credits and fee exemptions. These products are designated 'orphan products' by the FDA's Office of Orphan Products Development, and if shown to be safe and effective can be approved for marketing. As of 20 July 2009, 343 orphan products have been marketed to treat rare diseases ${ }^{3}$. By contrast, only 10 such products were approved in the 10 years preceding the $\mathrm{ODA}^{4}$.

The ODA has been credited not only with offering new hope for those who suffer from rare diseases, but also with nurturing an expanding biotechnology sector and personalizing drug development ${ }^{5,6}$. In a time of relative contraction in the development of new small-molecule therapeutics, orphan drug development has emerged as a burgeoning sector of the pharmaceutical industry. This correspondence documents its growth.

We used internal and publicly available data on first-time FDA approvals ${ }^{3,7}$ from January 1983 through to December 2008. We categorized approvals as 'drugs' if marketing authorization was gained under a New Drug Application (NDA) or as 'biologics' if under a Biologics License Application (BLA). We further classified these approvals as 'orphan' or 'non-orphan', depending on whether the product held an antecedent orphan designation. Only new drugs and biologics were considered; we did not include efficacy supplements (applications for new indications of already approved drugs) or formulation changes.

In 1983, the year when the ODA was signed, there was 1 new orphan drug approval and no new orphan biologic approvals. FIG. 1 shows trends by 5 -year periods for all 636 new drugs and 123 new biologics that were approved by the FDA between 1984 and 2008. Trends for orphan drug and biologic approvals were different. FIG. 1a shows that the number of orphan drugs approved remained relatively constant from 1984 through 2008 (with 31 approvals
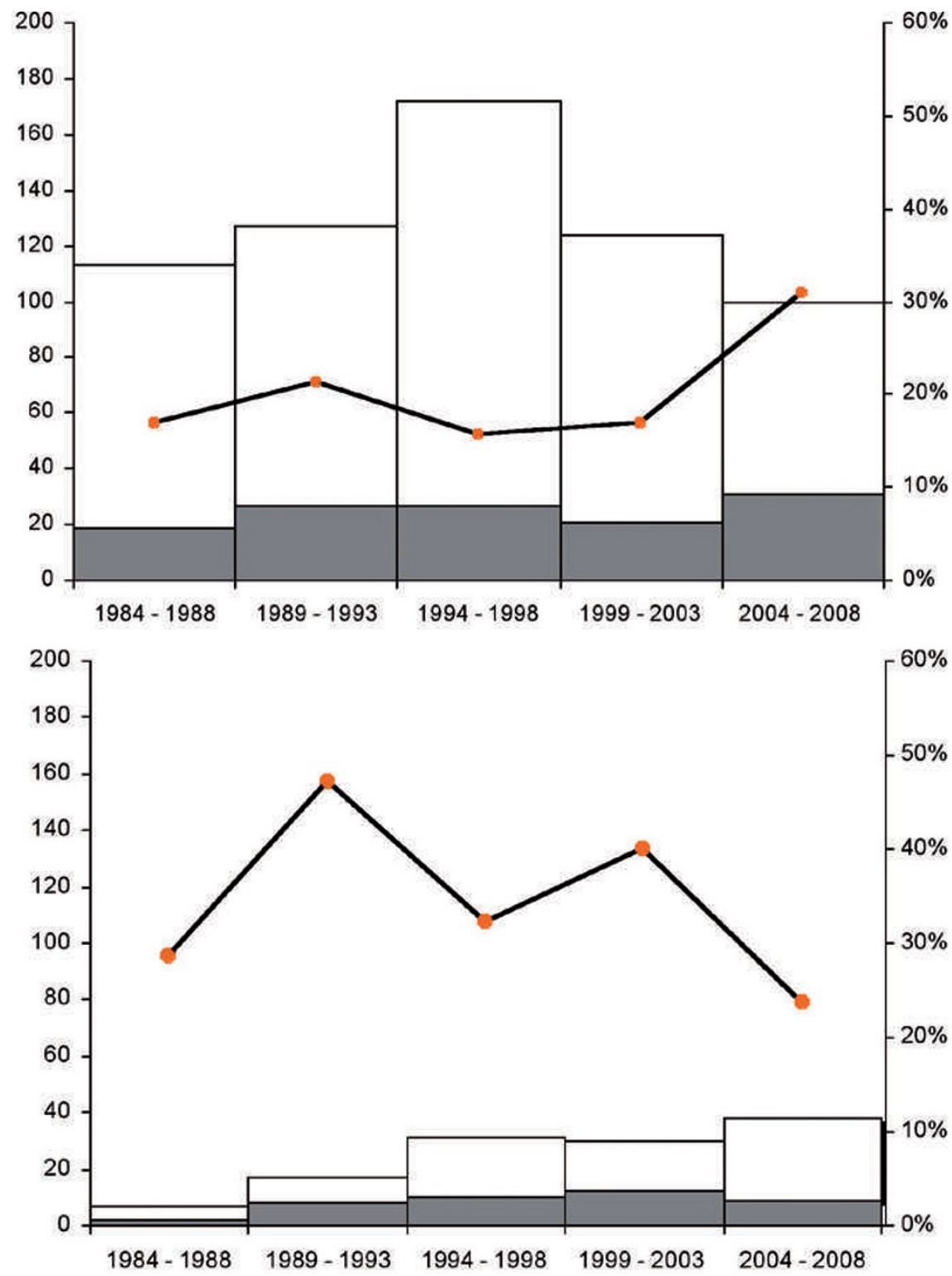

Figure 1 | Orphan and non-orphan new drugs and biologics approved by the FDA from 1984 to 2008. a | New drug approvals. Bar graph represents total number of orphan (dark bar at bottom) and non-orphan (white bar at top) new drugs approved every 5 years. Line graph represents percentage of new drug approvals that were orphan drugs in each 5-year period. b| New biologics approvals. Bar graph represents total number of orphan (dark bar at bottom) and non-orphan (white bar at top) new biologics approved every 5 years. Line graph represents percentage of new biologic approvals that were orphan biologics in each 5-year period. 


\section{CORRESPONDENCE}

In summary, orphan products now represent roughly one-third of all FDA newly approved drugs and biologics. As pharmacogenomics increasingly resolves specific therapeutic targets, we expect more orphan treatments for people with rare diseases. There are at least 6,000 rare diseases that collectively affect 25 million Americans ${ }^{8}$, and these trends hold promise for future therapies for such diseases.

Timothy Coté, Aditya Kelkar, Kui Xu and M. Miles Braun are at the Food and Drug Administration, Office of Orphan Products Development, Rockville, Maryland 20857, USA.

M. Ian Phillips and Aditya Kelkar are at the Keck Graduate Institute, Claremont, California 91711, USA.

Correspondence to T. C. e-mail: timothy.cote@fda.hhs.gov

doi: 10.1038/nrd2546-c1
Hughes, B. Rare incentives. Nature Rev. Drug Discov. 7, 190-191 (2008).

2. Food and Drug Administration. Orphan Drug Act. FDA website [online], < http://www.fda.gov/ForIndustry/ DevelopingProductsforRareDiseasesConditions/ Overview/ucm 119477.htm >

3. Food and Drug Administration. Search Orphan Drug Designations and Approvals. FDA website [online], $<$ http://www.accessdata.fda.gov/scripts/opdlisting/ oopd/index.cfm>

4. Haffner, M. E. Adopting orphan drugs-two dozen years of treating rare diseases. N. Engl. J. Med. 354 445-477 (2006)

5. The US Orphan Drug Act celebrates its 25th Anniversary. Eurodis website [online], $<$ http://www.eurordis.org/article.php3?id article $=1751>$ (2008).

6. Yin, W. Market incentives and pharmaceutical innovation. J. Health Econ. 27, 1060-1077 (2008).

7. NME Drug and New Biologic Approvals. FDA website [online], < http://www.fda.gov/Drugs/ DevelopmentApprovalProcess/

HowDrugsareDevelopedandApproved/ DrugandBiologicApprovalReports/ucm 121136.htm >

8. National Organization for Rare Diseases

About NORD. NORD website [online],

$<$ http://nord-rdb.com/info/about.html $>$. 